\title{
Totally Endoscopic Septal Myectomy in a Patient with Aortic Prothesis and Complicated with Lymphoma
}

\author{
Huimin $\mathrm{Cui}^{1}$, Lin Zhang ${ }^{2}$, Shixiong $\mathrm{Wei}^{3}$, and Shengli Jiang ${ }^{1}$ \\ ${ }^{1}$ Affiliation not available \\ ${ }^{2}$ the Chinese PLA General Hospital \\ ${ }^{3}$ Chinese PLA General Hospital
}

December 27, 2020

\begin{abstract}
Abstract The Morrow procedure which uses a median sternotomy with the left ventricle and septum accessed by the aortic valve through a vertical aortotomy is the current standard procedure for left ventricular outflow tract obstruction in hypertrophic cardiomyopathy. However, this approach may be limited in patients who have previously undergone aortic valve replacement. Here, we present a patient who complicated with lymphoma underwent endoscopic septal myectomy to preserve the previously implanted aortic prosthesis. Keywords: Endoscopic surgery, hypertrophic cardiomyopathy, septal myectomy.
\end{abstract}

\section{Introduction}

Hypertrophic cardiomyopathy is as an autosomal dominant cardiac sarcomere protein gene abnormality with a prevalence of $1 / 500$ in the general population [1]. It can create severe left ventricular outflow tract (LVOT) obstruction, which often results in a high sub-aortic pressure gradient with potentially lethal symptoms including sudden cardiac death, arrhythmias, and heart failure [2]. In 1964, Morrow developed a trans-aortic approach to resect a large part of the ventricular septum to enlarge the LVOT, which has remained the gold standard for surgically treating this pathology [3]. The potential limitations of the procedure include difficulties with visualizing the surgical anatomy and risks of aortic valve damage and an aortotomy [4], which would be particularly manifest when performed in redo-cardiac surgery. Here, we present a case of endoscopic septal myectomy to preserve the previously implanted aortic prosthesis in a patient who complicated with lymphoma.

\section{Case presentation}

A 65-year-old female who was diagnosed as lymphoma one months ago developed progressive dyspnea during chemotherapy. Inquiring about her past medical history, the patient had undergone a $23 \mathrm{~mm}$ St. Jude mechanical aortic valve replacement (AVR) 8 years ago. Preoperative echocardiogram reveled hypertrophic obstructive cardiomyopathy (HOCM) with a left ventricular posterior wall and maximum septal thickness measured at $16 \mathrm{~mm}$ and $23 \mathrm{~mm}$ respectively. Hypertrophic anterior papillary muscle and systolic anterior motion of the mitral valve was observed with an LVOT gradient measured at $239 \mathrm{mmHg}$ (Fig.1, A).

An endoscopic septal myectomy on the beating heart was planned. The cardiopulmonary bypass and the surgical approach was established as the conventional endoscopic mitral surgery (Fig.2, A). The LVOT was exposed through mitral orifice by resecting its anterior leaflet (Fig.2, B), however, it failed for large amounts of blood caused by aortic prothesis insufficiency owing to left atrial retractor (Fig.2, C). Aortic occlusion was accomplished by placing Chitwood clamp and cardiac arrest was established with antegrade custodiol cardioplegia after dividing ascending aorta (Fig.2, D). With the excellent surgical visualization 
obtained by endoscopy, we firstly resect the hypertrophic anterior papillary muscle (Fig.2, E), then the hypertrophied interventricular septum (Fig.2, F), and finally the bulging subaortic septum. Some site-specific hypertrophic muscle especially located under aortic prothesis which was difficult to resect was cauterized with electrocoagulation (Fig.2, G). Finally, a $25 \mathrm{~mm}$ St. Jude mechanical mitral valve was implanted (Fig.2, $\mathrm{H})$. Intraoperative transesophageal echocardiogram showed the septal thickness was $13 \mathrm{~mm}$ with an LVOT gradient of 4mmHg (Fig.1, B). At her 6-month follow-up, we noted no significant intracavitary gradient with both prostheses in good condition. The cardiac function was fully tolerant during her later chemotherapy.

\section{Discussion}

The traditional approach of myectomy which described septal exposure by way of a left atriotomy and takedown of the anterior mitral leaflet for symptomatic patients with LVOT obstruction is abandoned for the current standard Morrow procedure given the challenge of septal visualization [4]. Meanwhile, the continuous evolution in endoscopic, robotic, and trans-catheter cardiac interventions has resulted in innovative techniques that simultaneously address LVOT obstruction and concomitant atrioventricular valve pathology in the context of HOCM [5].

Adoption of an "endoscopic" approach provides direct and targeted access to the LVOT, the majority of the interventricular septum, and the mitral valve [5]. This is facilitated to incise the hypertrophied interventricular septum and provide the possibility to eliminate site-specific hypertrophic muscle bands such as subaortic septum. The superiority may be more evident in robotic surgery, but it has been used in only few cardiac centers.

Reoperation for LVOT obstruction in patients who have previously undergone a mechanical AVR is particularly challenging. The standard Morrow approach through transaortic access must remove the previous aortic prothesis at first and implant another new prothesis at the end of the procedure. Moreover, suboptimal surgical exposure of site-specific hypertrophic cardiomyopathy would be magnified for the relatively fixed cardiac position caused by extensive adhesion. Even if adhesions could be divided, the increased trauma, added blood loss and prolonged operation time, would result in greater impairment on cardiac function and prolonged postoperative recovery. In addition, even if the LVOT septum resection is satisfied, the intervention of the mitral would be another problem. A right lateral transmitral approach would also be limited by inadequate exposure of the bulging subaortic septum. Finally, it is extremely important to minimize the damage due to the complicated lymphoma in this case.

Considering the essence of modern surgery of minimizing the damage, individualized treatment for different cases is beneficial to postoperative recovery, especially in complex redo-cardiac surgery.

\section{Reference}

1. Maron BJ. Hypertrophic cardiomyopathy: an important global disease. Am J Med. 2004; 116: 63-5.

2. Maron BJ, Maron MS. Hypertrophic cardiomyopathy. Lancet. 2013; 381:242-255.

3. Chitwood WR. Robotic trans-atrial and trans-mitral ventricular septal resection. Ann Cardiothorac Surg 2017; 6: 54-59.

4. Khalpey Z, Korovin L, Chitwood WR, et al. Robot-assisted septal myectomy for hypertrophic cardiomyopathy with left ventricular outflow tract obstruction. J Thorac Cardiovasc Surg 2014; 147: 1708-1709.

5. van der Merwe J, Casselman F, Van Praet F. Endoscopic Port Access ${ }^{\mathrm{TM}}$ left ventricle outflow tract resection and atrioventricular valve surgery. J Vis Surg 2018;4:100.

\section{Figure Legends:}

Figure.1 A) Preoperative echocardiogram reveled hypertrophic obstructive cardiomyopathy and systolic anterior motion (arrow) with a pressure gradient of $239 \mathrm{mmHg}$. B). Intraoperative transesophageal echocardiogram showed the septal thickness was $13 \mathrm{~mm}$ with a pressure gradient of $4 \mathrm{mmHg}$ after resection.

Figure.2 A) The surgical approach was established as the conventional endoscopic mitral surgery. B) 
Resection of mitral anterior leaflet. C) Large amounts of blood in left ventricular. D) Cardiac arrest was established after ascending aorta separation and occlusion. E) Resection of the hypertrophic anterior papillary muscle. F) Resection of the the hypertrophied interventricular septum. G) Hypertrophic muscle located under aortic prothesis (arrow) was cauterized. H). A mechanical mitral prothesis was implanted.
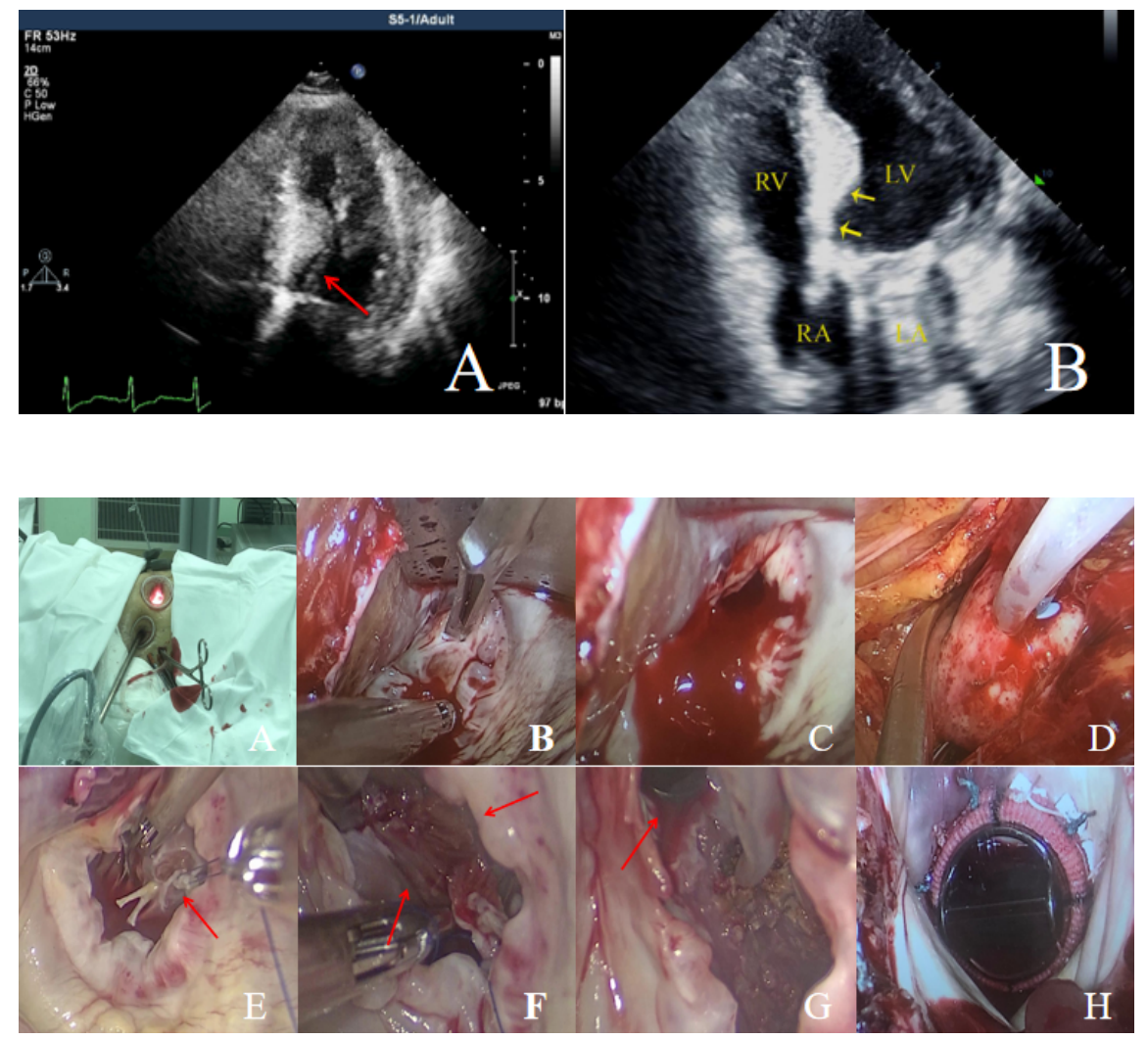